\title{
Correlations and fractality in random Ising magnets
}

\author{
U. Nowak ${ }^{1}$ and K.D. Usadel \\ Theoretische Tieftemperaturphysik, Universität Duisburg, Lotharstr. 1, W-4100 Duisburg 1, \\ Germany
}

\begin{abstract}
We investigate the behavior of domains in random Ising magnets by a Monte Carlo simulation. We calculate the spatial correlation function of the frozen domain state of twoand three-dimensional diluted antiferromagnets in an external magnetic field and compare the results with those line shapes found from neutron scattering experiments. An analysis leads to a new interpretation of the corresponding correlation length in terms of fractality and interpenetration in the structure of the domains.
\end{abstract}

Diluted Ising antiferromagnets in an external magnetic field (DAFF) are believed to be in the same universality class like the random field Ising model (RFIM) [1]. A well known feature of the DAFF is the formation of a domain state with extremely long relaxation times. This domain state is thought to be metastable for lower fields and it is obtained by cooling the system in an external field from the paramagnetic high temperature phase. From neutron scattering experiments it was concluded that this field cooled state is frozen at low temperatures since the correlation length, which is often thought to be a typical domain radius, remains finite [2]. The mechanisms which are responsible for the corresponding hysteretic properties of the DAFF have been investigated experimentally [2,3], theoretically [4] and in computer simulations [5-7]. Here we want to focus on the structural properties of the frozen domains in both two and three-dimensional systems.

The Hamiltonian of the DAFF in units of the coupling constant $J$ reads

$$
H=\sum_{\langle i, j\rangle} \varepsilon_{i} \varepsilon_{i} \sigma_{i} \sigma_{i}-B \sum_{i} \varepsilon_{i} \sigma_{i},
$$

where $\sigma_{i}= \pm 1$ and $\varepsilon_{i}=0.1$.

We considered nearest neighbor interaction only and used helical boundary conditions and the heat-bath algorithm. Typical sizes of the simple cubic, 
respectively square lattices were $99 \times 99 \times 98$ and $199 \times 198$ but even larger lattices with sizes of up to $145 \times 145 \times 144$ and $999 \times 998$ have been investigated in order to confirm our findings on larger length scales. The dilution $p$ was $50 \%(3 \mathrm{D})$ and $25 \%(2 \mathrm{D})$.

We used a completely random distribution of spins as the initial configuration, corresponding to a paramagnetic state. Then we cooled the system in the field $B$ by lowering the temperature step by step. A domain state develops, the structure of which depends on the cooling rate, i.e. on the number of Monte Carlo steps per spin (MCS) per temperature step. By cooling the system slowly enough a practically frozen domain state can be reached. This domain state is metastable in the sense that there is no observable development towards antiferromagnetic long range order.

We determined the correlation function $c(r)$ directly. E.g. in three dimensions the correlation function is the average correlation of spins with perpendicular distance $r$ from the mean plane of the system with the spins in this plane. Periodic as well as free boundary conditions have been employed in this direction in order to investigate the influence of the surface of the finite lattice. We found that confining the determination of $c(r)$ to small enough $r<L / 2$, where $L$ is the linear system size, we can neglect the influence of boundary conditions.

Fig. 1 shows a semi-logarithmic plot of $c(r)$ for three dimensions during field cooling. 25 systems with $L$ ranging from $L=99$ to 49 have been cooled in a field of $B=0.5$ in temperature steps $\Delta T=0.1$ from a temperature $T_{\max }=1.9$ to $T_{\min }=0.8$ with a cooling rate of $1000 \mathrm{MCS} / \Delta T$. The field cooling of the two-dimensional systems has been performed correspondingly, details will be published elsewhere.

In both, two and three dimensions a freezing of the system can be observed, meaning that the correlation length $\xi$, which is more or less the slope of the curves, remains constant. The correlation function in the critical region of the three-dimensional RFIM has the form $c(r)=c_{0} r^{-(d-4+\bar{\eta})} \exp (-r / \xi)$. Fitting our

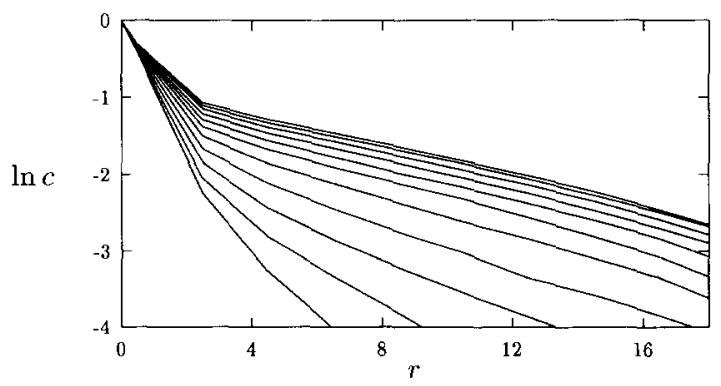

Fig. 1. In $c$ versus $r$ for different temperatures during field cooling (3D). 
data to this form results in exponents $d-4+\bar{\eta}$, which are typically less than 0.1 in both two and three dimensions. These very small power law corrections give evidence for the fact that $c(r)$ is well described by a pure exponential. This confirms the neutron scattering line shapes $I(q)$, which are the Fourier transform of $c(r)$, to be well fitted by a squared Lorentzian (3D) [8,9] and a Lorentzian to 1.5 (2D) [10], respectively. All these findings are in agreement with the experimental studies. We conclude that the correlations of the frozen domain state behave like those in the critical region, with $\bar{\eta} \approx d-2$, which is also in agreement with theoretical studies of the critical behavior of the RFIM [11].

Neglecting the small power law corrections we determined $\xi$ for different fields by directly fitting to a pure exponential. In the two-dimensional case a simple power law holds for slow enough cooling rates. Fitting yields $\xi \sim$ $B^{-1.52 \pm 0.04}$ in agreement with the experimental finding $\xi \sim B^{-1.6}[10]$. In three dimensions and in the limit of high fields the corresponding power law relation is $\xi \sim B^{-2.3}$. For lower field $\xi$ depends on the cooling rate, an effect which hardly could be overcome in our simulation. However, the high field limit of the exponent is in agreement with the experimental findings $\xi \sim B^{-2.2 \pm 0.1}$ (see ref. [9] and references therein).

The correlation lengths in our simulations are ranging from about 3 to about 18. The corresponding correlation lengths determined through neutron scattering are ranging from 1 to 240 [12] (all lengths cited here are measured in units of the lattice constant). Surprisingly, the experimental results in the limit of high fields and high dilution and our results are of the same order of magnitude even though experimental systems are much larger, of course, than ours. This, together with the fact that the scaling of $\xi$ with $B$ coincides with experimental results shows that the structural findings of our simulations are relevant for the length scales in experimental systems as well.

Now we want to focus on the question how to interpret the correlation length microscopically. Fig. 2 shows the 2D domain state arising after field cooling in a field of $B=1.5$ with a cooling rate of $5000 \mathrm{MCS} / \Delta T$. The corresponding correlation length is about 3.5. Since fig. 2 represents a $399 \times$ 398 system this is less than $1 \%$ of the linear lattice size and therefore certainly not the radius of the largest domain, which has a volume of 22396 spins corresponding to a radius of about 148 . Also, we found numerically that $\xi$ is not a mean or typical radius. Due to the fractality of the domains $[7,13]$ the structure is self-similar on all occurring length scales. Therefore, there is a broad spectrum of domain sizes in the system and no typical length scale. We conclude that $\xi$ is a length scale which is not related to the size of domains. This is rather surprising since near a critical point the mean radius of clusters has been found to be proportional to the correlation length [14]. However, the 


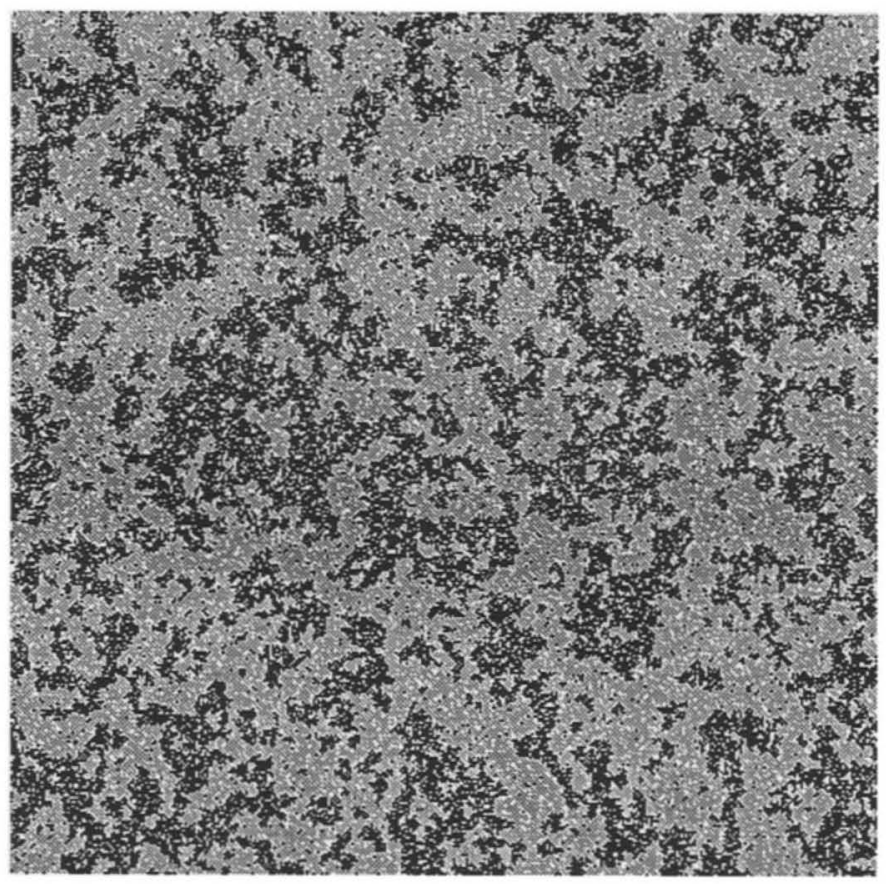

Fig. 2. Frozen domain state of a $399 \times 398$ system. The two phases are represented in black and grey. Vacancies are not shown.

system we consider is not at the critical point and due to the freezing it consists mainly of rather large interpenetrating domains. Due to this unusual behavior, we interpreted the correlation length as being related to the interpenetration of the domains [7,12], i.e. with the number of phase changes one undergoes walking through the system.

It should be noted that these findings contradict assumptions which are often made for domains, clusters, droplets or related objects, since these are normally thought to be compact without domains inside domains fulfilling Euclidean scaling relations. In this picture it may be useful to imagine the correlation length as a "typical domain radius" but we suggest to consider the correlation length of the DAFF rather as a "mean interpenetration length".

\section{References}

[1] S. Fishman and A. Aharony, J. Phys. C 12 (1979) L729.

[2] R.J. Birgeneau, R.A. Cowley, G. Shirane and H. Yoshizawa, J. Stat. Phys. 34 (1984) 817; D.P. Belanger, S.M. Rezende, A.R. King and V. Jaccarino, J. Appl. Phys. 57 (1985) 3294. 
[3] U.A. Leitão, W. Kleemann and I.B. Ferreira, Phys. Rev. B 38 (1988) 4765;

P. Pollak, W. Kleemann and D.P. Belanger, Phys. Rev. B 38 (1988) 4773.

[4] J. Villain, Phys. Rev. Lett. 52 (1984) 1543.

[5] G.S. Grest, C.M. Soukoulis and K. Levin, Phys. Rev. B 33 (1986) 7659.

[6] U. Nowak and K.D. Usadel, Phys. Rev. B 39 (1989) 2516.

[7] U. Nowak and K.D. Usadel, Phys. Rev. B 44 (1991) 7426.

[8] D.P. Belanger, A.R. King and V. Jaccarino, Phys. Rev. B 31 (1985) 4538.

[9] H. Yoshizawa, R.A. Cowley, G. Shirane and R. Birgeneau, Phys. Rev. B 31 (1985) 4548.

[10] H. Yoshizawa, R.A. Cowley, G. Shirane, R.J. Birgeneau, H.J. Guggenheim and H. Ikeda, Phys. Rev. Lett. 48 (1982) 438.

[11] A.J. Bray and M.A. Moore, J. Phys. C 18 (1985) L927.

[12] R.A. Cowley, H. Yoshizawa, G. Shirane and R.J. Birgeneau, Z. Phys. B 58 (1984) 15.

[13] U. Nowak and K.D. Usadel, Phys. Rev. B, to be published.

[14] J.-S. Wang and D. Stauffer, Z. Phys. B 78 (1990) 145. 